\title{
THE BURDEN ON A UROLOGIST OF PERCUTANEOUS NEPHROSTOMIES AND ANTEGRADE URETERIC STENTS: SHOULD TRAINEE UROLOGISTS LEARN TO PERFORM THESE PROCEDURES?
}

\author{
Jack Donati-Bourne $\mathrm{J}^{1}$, Shahd Seifeldin Nour ${ }^{2}$, Zain Siddiqui ${ }^{3}$, Richard P. Viney RP \\ ${ }^{1}$ ST6 Urology, Urology Department, University Hospitals Birmingham NHS Foundation Trust, UK \\ ${ }^{2}$ FY2 Doctor, Emergency Department, University Hospitals North Midlands NHS Trust, UK. \\ ${ }^{3}$ CT1 Urology, Urology Department, George Eliot Hospital NHS Trust, UK \\ ${ }^{4}$ Consultant Urologist, Urology Department, University Hospitals Birmingham NHS Foundation Trust, UK.
}

Corresponding author: dr.donatibourne@gmail.com

Submitted: September 1, 2019. Accepted: November 7, 2019. Published: November 28, 2019.

\begin{abstract}
Background and Objective

Renal obstruction is a common urological emergency potentially requiring urgent decompression by percutaneous nephrostomy $(\mathrm{PCN})$ or antegrade ureteric stent (AUrS), procedures both performed by interventional radiologists, or retrograde stenting in theatre by a urologist.

The study aimed to assess the burden of emergency PCN/AUrS on the overall workload of a urology department and evaluate the impact of procedural delays in terms of bed-occupancy and cost.

The findings serve to explore whether formal PCN/AUrS training would be desirable for UK trainees in urology.
\end{abstract}

\section{Material and Methods}

A prospective study of all patients admitted under urology at Queen Elizabeth Hospital Birmingham (QEHB) between $20^{\text {th }}$ October $-18^{\text {th }}$ November 2018.

Electronic records to retrieve data about admission, treatment provided, length of in-patient stay, length and reason for delay awaiting PCN/AUrS, including use of anti-coagulants.

\section{Results}

$\mathrm{n}=148$ patients identified. $\mathrm{n}=22(14.8 \%$ of total $)$ primary admission reason and/or main treatment provided related to PCN/AUrS. 601 urology in-patient days occupied for all causes, $166(27.6 \%)$ related to PCN/AUrS and 66 (10.9\%) awaiting PCN/AUrS (delays cost $£ 11,361 /$ month). 13 days (19.6\% of all delay days) were lost waiting for reversal of effects of anti-coagulant medication.

\section{Conclusion}

PCN/AUrS constituted a noteworthy proportion of all admissions and in-patient bed days in QEHB urology. Clinically non-urgent patients experienced notable cumulative delays whilst awaiting PCN/AUrS which adversely impacted bed occupancy. A suitably trained urologist competent at PCN/AUrS may positively address these issues. The findings merit consideration of a call for UK urology trainees to be trained in $\mathrm{PCN} / \mathrm{AUrS}$ as part of CCT requirements. 
Renal obstruction leading to loin pain, sepsis and/or renal failure is a common urological emergency. ${ }^{1}$ Options for emergency drainage include retrograde ureteric stent (RUrS) in theatre by a urologist or ultrasound (US) guided percutaneous nephrostomy tube (PCN) in the interventional radiology (IR) suite by a radiologist, plus/minus antegrade ureteric stent (AUrS). ${ }^{2}$

National Institute of Clinical Excellence (NICE) and European Association of Urology (EAU) 2019 guidelines (Level $1 \mathrm{~b}$ evidence) considers either RUrS or PCN acceptable interventions to treat an obstructed kidney and the decision lies with the attending urologist. ${ }^{3,4}$

Delays in PCN insertion may occur due to nonclinical factors such as local resources and staffing. Trusts lacking 24-hour on-site IR may necessitate temporary patient transfer for PCN insertion, and busy IR departments may not be able to promptly perform less urgent PCNs resulting in prolonging in-patient admissions. ${ }^{5}$

UK urological trainees are currently not required for Certification Completion Training (CCT) purposes to acquire formal skills in performing US, although there has previously been a call for integrating such skills in training schemes. ${ }^{6}$

In other countries such as the US and India, urologists routinely perform their PCN insertion. The benefits to the urologist of being proficient at PCN may include greater autonomy in emergency management with less reliance on IR, thus streamlining the obstructed kidney patient's journey. ${ }^{6}$

Drawbacks may include greater procedural risk if the urologist's PCN experience is not comparable to radiology colleague, furthermore, the added competency in a fixed duration training scheme may jeopardize acquisition of other urological skills.

The study aimed to assess the relative burden of emergency PCN/AUrS in the overall emergency workload of a tertiary referral urology department. The study also aimed to assess relative impact of delays waiting for emergency PCN/AUrS in terms of bed-occupancy and cost, as well as evaluating the reason for delay including pre-procedural use of anticoagulant medication.
The authors propose the findings should serve to generate further interest at a national level to formally evaluate whether PCN/AUrS training should become mandatory for UK trainees seeking CCT in urology.

\section{MATERIALS AND METHODS}

A single-centre prospective cohort study was undertaken within the urology department at the Queen Elizabeth Hospital Birmingham (QEHB) - a tertiary referral centre with 24-hour on-site IR service and a dedicated 36-bed urology ward.

All elective and emergency patients admitted under the care of the urology department between $0.00 \mathrm{am}$ of $20^{\text {th }}$ October 2018 and $23.59 \mathrm{pm}$ on $18^{\text {th }}$ November 2018 ( 30 consecutive days) were included in the study.

Elective planned attendances for scheduled PCN changes and/or AUrS insertions were excluded.

"Group of interest" (GOI) was defined as patients whose main reason for admission was a PCN-related problem (such as PCN blockage, infection or bleeding) and/or patients whose main definitive treatment provided during their admission was PCN insertion/ change and/or AUrS insertion.

QEHB Electronic records were used to collect the following data:

- Number of patients admitted and treated by the urology department for all reasons, and the total number of in-patient occupied urology bed days

- The number and clinical details of patients in GOI

- Number of in-patient occupied urology bed days by GOI

- Number of in-patient occupied urology bed days where main component of management plan was awaiting PCN and/or AUrS (calculated by lag time between electronic request and effective completion of the procedure)

- Reason for delay in performing PCN/AUrS including use of pre-procedural anti-coagulant medication

Data was also collected for comparative purposes to evaluate the number in-patient occupied urology bed days whose main reason for admission and stay were common urological procedures including transurethral resection of the prostate (TURP), transurethral 
resection of bladder tumour (TURBT) and pelvic oncology (prostatectomy, cystectomy) surgery.

The cost of an overnight in-patient urology bed at QEHB was obtained from the Trust's Finance department, and the figure used to estimate the economic burden to Trust attributable to waiting for PCN and/or AUrS.

\section{RESULTS}

In the study period, a total of $(\mathrm{n}=148)$ urological patients were admitted and treated on the urology ward for all causes. Of these $(n=22)(14.8 \%)$ were patients whose primary admission reason and/or main definitive treatment provided during admission was related to PCN and/or AUrS (GOI)

One patient in GOI was admitted and discharged twice during the study period with PCN-related cause. A combined total of 601 in-patient urology bed days were occupied for all urological causes (mean 4.1 days per patient). A combined total of 166 in-patient urology bed days were occupied for GOI (mean 7.5 days per patient) $(27.6 \%$ of total in-patient urology days). A total of 66 in-patient occupied urology bed days were recorded where the main component of the management plan was awaiting PCN insertion/ exchange or AUrS procedure (10.9\% of total urology bed days) (Table 1).

The waiting time was calculated starting from the moment the patient was deemed treated and stabilized (for example from sepsis) and thus ready for the required procedure, and ending at the time the procedure was performed.

The direct cost per urology bed day was $£ 172.14$, to include nursing and non-pay costs (consumables,

TABLE 1 Occupied Urology Bed Days

\begin{tabular}{|l|c|c|}
\hline & $\begin{array}{c}\text { Total Occupied } \\
\text { Urology Bed Days }\end{array}$ & \% of total \\
\hline All urology patients & 601 & \\
\hline GOI & 166 & 27.6 \\
\hline $\begin{array}{l}\text { GOI awaiting } \\
\text { PCN/AurS }\end{array}$ & 66 & 10.9 \\
\hline
\end{tabular}

$A U r S=$ antegrade ureteric stent; $G O I=$ group of interest; $P C N=$ percutaneous nephrostomy tube. drugs) but excluding the cost of attending doctors. The estimated cost burdened on QEHB Trust in terms of occupied urology bed days associated with waiting for such procedures was $£ 11,361$ per month (excluding costs for attending doctors).

30 urology bed days were occupied awaiting PCN insertion/exchange and 36 days awaiting AUrS. The most common reasons for GOI admission were obstructed kidney due to metastatic cancer and infected obstructed kidney due to urolithiasis (Table 2). All cases of acutely infected obstructed kidneys were successfully completed within 4 hours.

For GOI 18 patients required emergency PCN insertion (4 patients bilateral, 22.2\%), 2 required PCN exchange and 2 were subsequently cancelled for clinical reasons. Nine patients had subsequent AUrS $(50.0 \%)$ (7 as in-patient, 2 as out-patient) (Table 3).

The authors incidentally noted that 5 GOI patients died within 6 weeks of admission (23.8\%) and all were patients with known underlying metastatic cancer ( 3 prostatic, 1 bladder and 1 esophageal). Within the

TABLE 2 Reason for Admission within GOI

\begin{tabular}{|l|c|}
\hline \multicolumn{1}{|c|}{ Reason for GOI Admission } & $\begin{array}{c}\text { Number of } \\
\text { Patients }\end{array}$ \\
\hline Obstructed kidney due to malignancy & 10 \\
\hline $\begin{array}{l}\text { Infected obstructed kidney due to } \\
\text { stone }\end{array}$ & 7 \\
\hline $\begin{array}{l}\text { Blocked/dislodged PCN requiring } \\
\text { exchange }\end{array}$ & 2 \\
\hline $\begin{array}{l}\text { Obstructed kidney due to benign } \\
\text { pathology }\end{array}$ & 2 \\
\hline $\begin{array}{l}\text { Delayed diagnosis of ureteric injury } \\
\text { after hysterectomy }\end{array}$ & 1 \\
\hline
\end{tabular}

$G O I=$ group of interest PCN = percutaneous nephrostomy tube.

TABLE 3 Main Definitive In-patient Intervention Provided to GOI Patients

\begin{tabular}{|l|c|}
\hline \multicolumn{1}{|c|}{ In-patient GOI Intervention } & Number of Patients \\
\hline Uni-lateral PCN & 14 \\
\hline Bi-lateral PCN & 4 \\
\hline PCN Exchange & 2 \\
\hline AUrS & 7 \\
\hline
\end{tabular}

AUrS = antegrade ureteric stent; $G O I=$ group of interest $; P C N=$ percutaneous nephrostomy tube. 
TABLE 4 Comparison of Occupied Urology Bed Days with Other Common Operations

\begin{tabular}{|l|c|}
\hline $\begin{array}{c}\text { Main Admission Reason or } \\
\text { Treatment Given }\end{array}$ & $\begin{array}{c}\text { Number of occupied } \\
\text { urology bed days }\end{array}$ \\
\hline Group of interest & 166 \\
\hline Pelvic oncology & 68 \\
\hline TURBT & 46 \\
\hline TURP & 40 \\
\hline
\end{tabular}

$T U R P=$ transurethral resection of the prostate;

TURBT = transurethral resection of bladder tumour.

same time in the department, 68 urology bed days were occupied due to pelvic oncological procedures, 46 for TURBT and 40 for TURP (Table 4).

Pre-procedural anti-coagulant use delayed PCN/ AUrS insertion in 3 patients (13.6\% of those requiring procedure) -1 on clopidogrel ( 8 days), 1 on apixaban (3 days), 1 on warfarin ( 2 days) for a cumulative total of 13 days. Three patients (13.6\%) were taking aspirin, however, the procedure was not paused for reversal of this medication.

\section{DISCUSSION}

The finding that $14.8 \%$ of all urological patients' primary admission reason and/or main definitive treatment was related to PCN/AUrS, which occupied $27.6 \%$ of all urology in-patient bed days, denotes a noteworthy contribution of GOI patients relative to the entire workload.

Furthermore, a greater number of bed days related to GOI (166) compared to the combined total of the three selected common procedures: pelvic oncology (68), TURBT (46) and TURP (40).

The total of 66 in-patient bed days recorded where the main management component was awaiting PCN/AUrS (10.9\% of total) and the associated estimated monthly cost of $£ 11,361$ to the Trust for these delays (estimated $£ 136,000 /$ year) underlines a serious financial and bed-occupancy issue. In all patients experiencing delay, this was due to lack of free and available fully staffed interventional radiology list for addition of the PCN/AUrS.

For urgent but non-emergency PCN/AUrS insertions the interventional radiology department requested that patients remain in a hospital bed to await their expedited procedure, as discharge followed by an outpatient slot would lead to unacceptable time delays potentially compromising patient safety.

US is a core component of PCN insertion and the importance of incorporating US in urology training has been evaluated. Surange et al. ${ }^{7}$ assessed the benefit of emergency US by urology trainees in 111 referrals, reporting that US proved life-saving in 5, significantly influenced management in 11 but was misleading in 22 , nonetheless concluding US is a useful tool for urologists.

Talreja et al. ${ }^{8}$ assessed proportions of emergency urological interventions in their tertiary hospital in India, finding PCN the most common procedure $(32.8 \%)$. Evidence supporting the use of US by urological trainees has also been found in the out-patient clinic with diagnostic accuracies greater than $90 \%$. 9,10

Lee et al. ${ }^{11}$ analyzed whether US-guided access training may have long-term benefits by surveying 35 residents, who were asked whether they received such training and continued to perform percutaneous renal procedures, finding that those trained were significantly more likely to continue performing USguided procedures in their later career.

One of the main reasons for the reticence certain urologists have to perform PCN is the perceived notion that radiologists possess better equipment/skills. A study retrospectively comparing PCNLs where access was obtained either by a urologist or radiologist revealed comparable stone clearance rates, however, cases performed by urologists were more complex resulting in multi-tract PCNL. ${ }^{12}$

Several studies have compared outcomes between urologists and radiologists in percutaneous access in PCNL. A large study on behalf of BAUS ${ }^{13}$ reviewed 5211 PCNLs over 6 years concluding that favourable outcomes may be expected where access is obtained by either urologist or radiologist provided they have received appropriate training.

A series with 650 urologist-directed $\mathrm{PCNs}^{14}$ yielded comparable results in terms of success rates and complications with radiologist-directed PCNs in the UK nephrostomy audit. ${ }^{15}$ This conclusion has resulted in the issue of whether urologists should undertake their nephrostomies being previously raised. 
Masood et al. ${ }^{16}$ supported this and proposed a change in culture from urologists, radiologists and BAUS. In the decade since the article evidence of their proposed greater involvement is not visible. Arguably ensuring that urological trainees achieve sufficient exposure to PCN/AUrS may be challenging, however currently UK radiology trainees do not have specific indicative numbers in PCN/AUrS required for $\mathrm{CCT}^{17}$, rather they require successful completion of work-based assessments.

This study underlines not only the relevance of PCN in the daily workload of a urologist but also the financial implications that delays in treatment access may have for Trusts. To the authors' knowledge, this is the only study in the literature assessing the burden on a urology department of PCN/AUrS relative to all admissions as well as the impact delays have on bedoccupancy and cost.

The first study limitation is the absence of a RUrS sub-analysis - an intervention that in many cases is an alternative to PCN. In this department, most consultants prefer PCN to RUrS to treat an infected obstructed kidney, and the authors believe the RUrS-related workload is therefore considerably less than GOI.

A further limitation is the non-characterization of urgency. The authors observed that in situations of an immediate emergency, PCNs were performed within hours in the Trust. The study's main aim was to evaluate the burden on the urology department of PCN/AUrS, rather than assess the efficiency of the IR service.

Arguably if there were an urologist trained in PCN/AUrS, the less urgent procedures would be completed sooner thus expediting discharges. The concept of a dedicated "Interventional urology list" performing procedures such as PCN/AUrS has been explored by a London Hospital, revealing comparably successful outcomes and proposing improved local efficiency. ${ }^{18}$

A final limitation relates to the bed-occupancy calculation, which was not further qualified if discharge delays occurred for other reasons, but rather ascribed to the initial admission reason and/or treatment provided. This method however applied to all study patients.

The authors propose further work may include a similar analysis at the regional level, as smaller Trusts may experience further delays due to the absence of 24-hour IR cover, and such a study would provide a more global NHS perspective.

Were calls to incorporate PCN/AUrS into urological training seriously considered, further work would be required to determine the appropriate number to be undertaken for $\mathrm{CCT}$ and whether this would require extended training or sacrifice of other procedures on the $\mathrm{CCT}$ checklist.

\section{CONCLUSION}

The study has demonstrated that urological patients whose main reason for admission was a PCN-related problem and/or patients whose main definitive treatment provided during admission was $\mathrm{PCN}$ and/or AUrS constitute a noteworthy element of the total workload faced by the urologist in daily practice.

The authors believe this finding should prompt serious consideration that for training adult urologists competency in such procedures should become mandatory as part of CCT requirements. This may have a positive impact on expediting patient care as well as reducing delays experienced waiting for such procedures to be performed by pressured IR colleagues.

\section{ACKNOWLEDGEMENTS}

The authors wish to thank Dr. Flavia Donati and Dr. Yaman Adi for their assistance in the production of the manuscript.

\section{CONFLICT OF INTEREST}

None declared.

\section{REFERENCES}

1. Rukin N, Siddiqui Z, Chedgy E, et al. Trends in upper tract stone disease in England: evidence from the Hospital Episodes Statistics Database. Urologia Internationalis 2016;98(4):391-96.

2. de Sousa Morais N, Pereira J, Mota P, et al. Percutaneous nephrostomy vs ureteral stent for hydronephrosis secondary to ureteric calculi: impact on spontaneous stone passage and health-related quality of life-a prospective study. Urolithiasis. 2018.

3. NICE.org. Acute kidney injury: prevention, detection and management | Guidance and guidelines | NICE [Internet]. 2019 Available from: https://www.nice.org. uk/guidance/cg169 (2019, accessed 14 February 2019) 
4. Turk C, Skolarikos A, Neisius A, et al. EAU Guidelines on Urolithiasis. Arnhem, The Netherlands, European Association of Urology, 2019. Available at: https:// uroweb.org/wp-content/uploads/EAU-Guidelines-onUrolithiasis-2018-large-text.pdf

5. ElSheemy M, Shouman A, Shoukry A, et al. Ureteric stents vs percutaneous nephrostomy for initial urinary drainage in children with obstructive anuria and acute renal failure due to ureteric calculi: a prospective, randomised study. BJU Int 2014;115(3):473-79.

6. Dudderidge T, Kayes O. Urology led ultrasound services time to focus. BJU Int 2007;981-83.

7. Surange RS, Jeygopal NS, Chowdhury SD, et al. Bedside ultrasound: a useful tool for the on-call urologist? Int Urol Nephrol 2001;32(4):591-6.

8. Talreja S, Banerjee I, Teli R, et al. A Spectrum of urological emergency reported at a tertiary care teaching hospital: an experience. J Clin Diag Res 2015;9(11):12.

9. Moslemi MK, Mahfoozi B. Urologist-operated ultrasound and its use in urological outpatient clinics. Patient Prefer Adher 2011;5:85.

10. Nargund VH, Cumming JA, Jerwood D, et al. Ultrasound in urological emergency: results of self audit and implications for training. Int Urol Nephrol 1996;28(3):267-71.

11. Lee C, Anderson JK, Monga M. Residency training in percutaneous renal access: does it affect urological practice? J Urol 2004;171(2):592-5.
12. El-Assmy AM, Shokeir AA, Mohsen T, et al. Renal access by urologist or radiologist for percutaneous nephrolithotomy-is it still an issue? J Urol 2007;178(3):916-20.

13. Armitage JN, Withington J, Fowler S, et al. BAUS section of endourology. Percutaneous nephrolithotomy access by urologist or interventional radiologist: practice and outcomes in the UK. BJU Int 2017;119(6):913-8.

14. Skolarikos A, Alivizatos G, Papatsoris A, et al. Ultrasoundguided percutaneous nephrostomy performed by urologists: 10-year experience. Urology 2006;68(3):495-9.

15. Chalmers N, Jones K, Drinkwater K, et al. The UK nephrostomy audit. Can a voluntary registry produce robust performance data? Clin Radiol 2008;63(8):888-94.

16. Masood J, Yeo L, Zaman F, et al. Should urologists in the UK undertake their own nephrostomies and renal access for endourological procedures: what does the future hold? BJU Int 2009;104(6):755-7.

17. The Royal College of Radiology Sub-Specialty Curriculum for Interventional Radiology. Available from: https://www.rcr.ac.uk/sites/default/files/ir_curriculum-2016_final_15_november_2016.pdf [last accessed 12 May 2019]

18. Masood J, Ismail M, El-Husseiny T, et al. 'An interventional urology list'-a novel concept for UK urological services. Ann R Coll Surg Engl 2010;93(1):27-30. 